\title{
The Inconsistent Data on the Effect of Vitamin D on Muscle Function
}

\section{Giulio Pioli ${ }^{1 *}$ and Andrea Giusti ${ }^{2}$}

${ }^{1}$ Geriatrics Unit, Department of Neuromotor Physiology, ASMN-IRCCS, Reggio Emilia, Italy

${ }^{2}$ Bone Clinic, Department of Gerontology and Musculoskeletal Sciences, Galliera Hospital, Genoa, Italy

Proximal myopathy and muscular weakness are one of the hallmarks of severe osteomalacia, together with the defective mineralization of newly formed bone matrix and bone pain [1]. Muscles biopsy studies have demonstrated that prolonged Vitamin D deficiency may produce a selective atrophy of type 2 muscular fibers, which are characterized by short, fast bursts of power and rapid fatigue [1].

The muscular tissue has a high specific nuclear receptor for 1,25-dihydroxyVitamin D [1,25(OH)2D], the active form of Vitamin D. In animal models, the presence of a non-functioning Vitamin D receptor (VDR) has been associated with subnormal muscle fibers, which presented with a diameter about $20 \%$ smaller [2].

Observational studies investigating differences in physical performances between patients with different levels of serum 25 -hydroxy-Vitamin D [25(OH)D], the precursor of 1,25(OH)2D and the most suitable indicator of Vitamin D status, found very inconsistent results. Marantes et al. [3] evaluated muscle mass and strength (e.g. hand grip) according to the $25(\mathrm{OH}) \mathrm{D}$ level in a large cohort of community-dwelling adults. They did not find any difference in the parameters assessed between quartiles of $25(\mathrm{OH}) \mathrm{D}$, even when they compared subjects with more severe Vitamin D deficiency [25(OH) $\mathrm{D}<10 \mathrm{ng} / \mathrm{ml}$ and controls [3].

Consistently with observational studies, randomized-controlled trials (RCTs) specifically designed to improve muscle performances trough Vitamin D supplementation/therapy led to contradictory results. In a systematic review published about 10 years ago, Latham [4] considered 13 trials undertaken to evaluate the effects of plain Vitamin D or its metabolites on physical function [4]. Ten studies did not demonstrate any positive result, while three showed a beneficial effect of Vitamin D combined with calcium on physical function [4]. In a more recent meta-analysis, Muir et al. [5] found that Vitamin D supplementation is capable of improving balance tests, producing, for example, a reduction of postural sway, but they failed to prove a valuable effect on lower extremity strength [5].

These inconsistencies arising from published observational and intervention studies may suggest that when administered to the general population Vitamin D has a poor and limited effect on muscle performances.

Bischoff-Ferrari et al. [6] has recently demonstrated that Vitamin D supplementation significantly reduces the risk of fracture only in those subjects receiving at least $800 \mathrm{IU}$ per day [6]. A threshold level of efficacy for cholecalciferol daily dose and, consequently, for the serum $25(\mathrm{OH}) \mathrm{D}$ value achieved is likely for any action of Vitamin $\mathrm{D}$, including muscle strength and function. Thus, the studies using low doses of Vitamin D (at least lower than 800 IU per day) could not be able to detect any beneficial effect of cholecalciferol supplementation just because of an inadequate intake, and due to failure in achieving the threshold for serum 25(OH)D in the majority of studied patients [7-9].

Notably, the relationship between serum 25(OH)D and muscle performances seems to be represented by a plateau shape rather than a linear one. In the Longitudinal Aging Study Amsterdam, the strength of this relationship leveled off for values of serum 25(OH)D above 30 $\mathrm{ng} / \mathrm{ml} \mathrm{[10],} \mathrm{indicating} \mathrm{that} \mathrm{it} \mathrm{is} \mathrm{unlikely} \mathrm{any} \mathrm{further} \mathrm{improvement} \mathrm{of}$ muscle strength for serum 25(OH)D levels above this limit of $30 \mathrm{ng} / \mathrm{ml}$.
These observations could, therefore, explain the reason why Vitamin D supplementation produced no significant effect in RCTs that enrolled patients with a mean basal serum $25(\mathrm{OH}) \mathrm{D}$ value close to the reference range [11-13]. On the other hand, it seems that improvements in muscle strength and function may be easily induced by plain Vitamin D supplementation in those subjects with Vitamin D deficiency, simply by contrasting the detrimental effects of deficiency on the muscles. Overall, these observations suggest that Vitamin D has just a physiologic role for muscle cells function rather than pharmacological effects.

Another relevant factor that may contribute to explain the huge variability in the results of RCTs is the choice of the test used to evaluate muscle performances. As Vitamin D seems to affect selectively the type 2 muscular fibers, the tools more sensitive and appropriate to define its beneficial effects should be those tests evaluating quick and short movements. For this reason, balance sway resulted, in general, positively influenced by Vitamin D supplementation, while handgrip or leg strength that needs more prolonged contraction gave more variable and inconsistent results.

Finally some recent reports described a relationship between muscle strength and some VDR polymorphisms [14]. Even if these data warrant further confirmation, an increase of serum $25(\mathrm{OH}) \mathrm{D}$ level could have different effects according to the VDR genotype (e.g. associated to higher or lower muscle power).

Summarizing, the effects of Vitamin D supplementation on muscle function and strength still need to be investigated in high quality RCTs taking into account all the variables above described potentially affecting the results. In particular, these studies should enroll only patients with very low serum 25 OHD values, should provide an adequate intake of cholecalciferol capable to normalize serum 25(OH)D $(>30$ $\mathrm{ng} / \mathrm{ml}$ ), and should assess muscle function using tests investigating quick movements rather than endurance. Finally, an analysis on VDR genotype should be provided to better interpret the final results.

\section{Acknowledgment}

Giulio Pioli declares no financial relationships with any organizations that might have an interest in the submitted work. Andrea Giusti is a member of the Advisory Board of the Italian Society of Osteoporosis, Mineral Metabolism and Skeletal Diseases (SIOMMMS) and he has received consulting fees from Novartis, Procter and Gamble, Eli Lilly and InFoMed (ECM provider, Milano, Italy).

\section{References}

1. Yoshikawa S, Nakamura T, Tanabe H, Imamura T (1979) Osteomalacic myopathy. Endocrinol Jpn 26: 65-72.

*Corresponding author: Giulio Pioli, Geriatrics Unit, Department of Neuromoto Physiology, ASMN-IRCCS, Viale Risorgimento, 80, 42100 Reggio Emilia, Italy, Tel: +39-0522206188; Fax: +39-0522206122; E-mail: giulio.pioli@asmn.re.it

Received October 10, 2012; Accepted October 11, 2012; Published October 13 2012

Citation: Pioli G, Giusti A (2012) The Inconsistent Data on the Effect of Vitamin D on Muscle Function. J Spine 1:e107. doi:10.4172/2165-7939.1000e107

Copyright: (c) 2012 Pioli G, et al. This is an open-access article distributed under the terms of the Creative Commons Attribution License, which permits unrestricted use, distribution, and reproduction in any medium, provided the original author and source are credited. 
2. Endo I, Inoue D, Mitsui T, Umaki Y, Akaike M, et al. (2003) Deletion of vitamin $D$ receptor gene in mice results in abnormal skeletal muscle development with deregulated expression of myoregulatory transcription factors. Endocrinology 144: 5138-5144

3. Marantes I, Achenbach SJ, Atkinson EJ, Khosla S, Melton LJ 3rd, et al. (2011) Is vitamin $\mathrm{D}$ a determinant of muscle mass and strength? J Bone Miner Res 26: $2860-2871$

4. Latham NK, Anderson CS, Reid IR (2003) Effects of vitamin D supplementation on strength, physical performance, and falls in older persons: a systematic review. J Am Geriatr Soc 51: 1219-1226.

5. Muir SW, Montero-Odasso M (2011) Effect of vitamin D supplementation on muscle strength, gait and balance in older adults: a systematic review and meta-analysis. J Am Geriatr Soc 59: 2291-2300.

6. Bischoff-Ferrari HA, Willett WC, Orav EJ, Lips P, Meunier PJ, et al. (2012) A pooled analysis of vitamin $D$ dose requirements for fracture prevention. $N$ Engl J Med 367: 40-49.

7. Bunout D, Barrera G, Leiva L, Gattas V, de la Maza MP, et al. (2006) Effects of vitamin $D$ supplementation and exercise training on physical performance in Chilean vitamin D deficient elderly subjects. Exp Gerontol 41: 746-752.

8. Janssen HC, Samson MM, Verhaar HJ (2010) Muscle strength and mobility in vitamin D-insufficient female geriatric patients: a randomized controlled trial on vitamin D and calcium supplementation. Aging Clin Exp Res 22: 78-84.
9. Latham NK, Anderson CS, Lee A, Bennett DA, Moseley A, et al. (2003) A randomized, controlled trial of quadriceps resistance exercise and vitamin $D$ in frail older people: the Frailty Interventions Trial in Elderly Subjects (FITNESS). J Am Geriatr Soc 51: 291-299.

10. Wicherts IS, van Schoor NM, Boeke AJ, Visser M, Deeg DJ, et al. (2007) Vitamin $D$ status predicts physical performance and its decline in older persons. $\mathrm{J}$ Clin Endocrinol Metab 92: 2058-2065.

11. Grady D, Halloran B, Cummings S, Leveille S, Wells L, et al. (1991) 1,25-Dihydroxyvitamin D3 and muscle strength in the elderly: a randomized controlled trial. J Clin Endocrinol Metab 73: 1111-1117.

12. Kenny AM, Biskup B, Robbins B, Marcella G, Burleson JA (2003) Effects of vitamin $D$ supplementation on strength, physical function, and health perception in older, community-dwelling men. J Am Geriatr Soc 51: 1762-1767.

13. Glendenning $P$, Zhu K, Inderjeeth $C$, Howat $P$, Lewis JR, et al. (2011) Effects of three monthly oral $150,000 \mathrm{IU}$ cholecalciferol supplementation on falls, mobility and muscle strength in older postmenopausal women: a randomised controlled trial. J Bone Miner Res.

14. Barr R, Macdonald H, Stewart A, McGuigan F, Rogers A, et al. (2010) Association between vitamin $D$ receptor gene polymorphisms, falls, balance and muscle power: results from two independent studies (APOSS and OPUS). Osteoporos Int 21: 457-466. 